Check for updates

Cite this: Chem. Sci., 2018, 9, 6659

๑ All publication charges for this article have been paid for by the Royal Society of Chemistry

Received 11th January 2018

Accepted 12th June 2018

DOI: $10.1039 / \mathrm{c} 8 \mathrm{sc} 00171 \mathrm{e}$

rsc.li/chemical-science

\section{Theory for polariton-assisted remote energy transfert}

\author{
Matthew Du, (D) a Luis A. Martínez-Martínez, (D) ${ }^{a}$ Raphael F. Ribeiro, (D) ${ }^{a}$ \\ Zixuan Hu, (D) binod M. Menon (D) de and Joel Yuen-Zhou (DD *a
}

\begin{abstract}
Strong-coupling between light and matter produces hybridized states (polaritons) whose delocalization and electromagnetic character allow for novel modifications in spectroscopy and chemical reactivity of molecular systems. Recent experiments have demonstrated remarkable distance-independent longrange energy transfer between molecules strongly coupled to optical microcavity modes. To shed light on the mechanism of this phenomenon, we present the first comprehensive theory of polariton-assisted remote energy transfer (PARET) based on strong-coupling of donor and/or acceptor chromophores to surface plasmons. Application of our theory demonstrates that PARET up to a micron is indeed possible. In particular, we report two regimes for PARET: in one case, strong-coupling to a single type of chromophore leads to transfer mediated largely by surface plasmons while in the other case, strongcoupling to both types of chromophores creates energy transfer pathways mediated by vibrational relaxation. Importantly, we highlight conditions under which coherence enhances or deteriorates these processes. For instance, while exclusive strong-coupling to donors can enhance transfer to acceptors, the reverse turns out not to be true. However, strong-coupling to acceptors can shift energy levels in a way that transfer from acceptors to donors can occur, thus yielding a chromophore role-reversal or "carnival effect". This theoretical study demonstrates the potential for confined electromagnetic fields to control and mediate PARET, thus opening doors to the design of remote mesoscale interactions between molecular systems.
\end{abstract}

\section{Introduction}

Enhancement of excitation energy transfer (EET) remains an exciting subfield of the chemical sciences. Although Förster resonance energy transfer ${ }^{\mathbf{1}}$ (FRET) is one of the most extensively studied and well known forms of EET, its efficiency range is only at 1-10 nm. ${ }^{2}$ As such, exploration of EET schemes beyond that of a traditional pair of donor and acceptor molecules has been a highly active area of research. For instance, the theory of multichromophoric FRET ${ }^{3}$ has been applied to demonstrate the role of coherent exciton delocalization in photosynthetic light harvesting. ${ }^{4-8}$ This coherence, which is due to excitonic

${ }^{a}$ Department of Chemistry and Biochemistry, University of California San Diego, La Jolla, California 92093, USA. E-mail: joelyuen@ucsd.edu

${ }^{b}$ Department of Chemistry, Department of Physics, Birck Nanotechnology Center, Purdue University, West Lafayette, IN 47907, USA

${ }^{c}$ Qatar Environment and Energy Research Institute, College of Science and Engineering, HBKU, Doha, Qatar

${ }^{d}$ Department of Physics, City College, City University of New York, New York 10031, USA

${ }^{e}$ Department of Physics, Graduate Center, City University of New York, New York 10016, USA

$\dagger$ Electronic supplementary information (ESI) available. See DOI: 10.1039/c8sc00171e coupling between molecular emitters, has also been argued to increase EET in mesoscopic multichromophoric assemblies, ${ }^{9}$ with recent studies even reporting micron-sized transfer ranges in H-aggregates. ${ }^{\mathbf{1 0 , 1 1}}$ Along similar lines, a well-studied process is plasmon-coupled resonance energy transfer, ${ }^{12}$ where molecules which are separated several tens to hundreds of nanometers apart can efficiently transfer energy between themselves due to the enhanced electromagnetic fields provided by the neighboring nanoparticles. ${ }^{\mathbf{1 3 , 1 4}}$ Transfer between molecules across even longer distances can be mediated by the in-plane propagation of surface plasmons (SPs), with micron ranges reported in the literature ${ }^{15,16}$ (see ref. 17 for an example of micronrange EET via other types of electromagnetic modes). Notably, the plasmonic effects in these last examples occur in the socalled weak-coupling regime, where the frequency of energy exchange between excitons and plasmons is much slower than their respective decays.

An intriguing advancement in PARET has recently been reported by the Ebbesen group for cyanine dye J-aggregates strongly coupled to a microcavity mode. ${ }^{18}$ For spatially separated slabs of donor and acceptor dyes placed between two mirrors, it was found that increasing the interslab spacing from 10 to $75 \mathrm{~nm}$ led to no change in the relaxation rate between the hybrid light-matter states or polaritons, thus revealing 
a remarkable distance independence of the process. Importantly, such PARET phenomenon was already noted in an earlier work by the Lidzey group ${ }^{19}$ with a different cyanine-dye system, although the interslab spacing was not systematically varied there; similar work was also previously reported for hybridization of Frenkel and Wannier-Mott excitons in an optical microcavity. ${ }^{20}$ Motivated by these experiments, we hereby present a quantum-mechanical theory for polariton-assisted energy transfer which aims to characterize the various types of PARET afforded by these hybrid light-matter systems. To be concrete, we do so within a model where the "photonic modes" are SPs in a metal film and consider spatially separated slabs of donors and acceptor dyes which electrostatically couple to one another as well as to the SPs. We present a comprehensive formalism which encompasses the cases where either one or both types of chromophores are strongly coupled to the SPs. We apply our theory to a model system similar to those reported by the Ebbesen and Lidzey groups. Our work complements recent studies proposing schemes to enhance one-dimensional exciton conductance. ${ }^{21,22}$ In those studies, the delocalization afforded by strong-coupling (SC) is exploited to overcome static disorder within the molecular aggregate. Here, the emphasis is not on disorder (surmountable also by polaritonic topological protection $^{23}$ ), but rather on PARET between two different types of chromophores, where energy harvested by one chromophore can be collected in another. This focus on long-range capabilities, as well as in-depth analyses of the rate contributions for the SC-induced states, provide fresh perspectives on PARET. In particular, we offer fascinating predictions for the experimentally unexplored scenario of "photonic modes" strongly coupled to one of donors or acceptors (the latter case was first theoretically investigated for the chromophores in a microcavity ${ }^{24}$ ).

As a preview, we highlight the structure and the main conclusions of this work (the latter are summarized in Table 1). We begin by introducing the general Hamiltonian for spatially separated slabs of donors and acceptor chromophores in Section 2. EET rates for a single or both types of chromophores strongly coupled to SPs are presented in Sections 2.1 and 2.2, respectively. In the former case, the rates are "FRET-like" 1 and shown to be dependent on spectral overlap (of absorption and emission lineshapes). ${ }^{25,26}$ For SC to donors, EET can be tuned for enhancement or suppression. This result is in stark contrast with that of acceptors-SPs SC where, surprisingly, EET to acceptor polariton states vanishes for large-enough samples. For the case when both chromophores strongly interact with SPs, transfer is instead mediated by vibrational relaxation in analogy with the theories of Davydov ${ }^{27,28}$ and Redfield. ${ }^{25,26,29}$ Nevertheless, PARET is still achieved. In Section 3, we apply the formalism to study a model system resembling cyanine dye Jaggregates. Our numerical simulations demonstrate that applying SC exclusively to donors enables PARET up to 1 micron. We also show that sufficiently intense SC to acceptors induces a "carnival effect" that reverses the role of the donor and acceptor. Lastly, when both chromophores are strongly coupled to SPs, we obtain sizable PARET rates at chromophoric separations over hundreds of nanometers which are in good agreement with experiments.

\section{Theory}

We begin by describing the polaritonic (plexcitonic) setup that we theoretically investigate. Let the chromophore slabs lie above $(z>0)$ and parallel to the metal film $(z<0)$ that sustains SP modes (example schematic diagrams are given in Fig. 1a, 2a, 3a, and 4a). We assume the metal film and the slabs are extended along the $x y$ (longitudinal) plane. The slabs of $\mathrm{C}=\mathrm{D}, \mathrm{A}$ (donor, acceptor) chromophores consist of $N_{x y, \mathrm{C}}, N_{z, \mathrm{C}}$, and $N_{\mathrm{C}}=N_{x y, \mathrm{C}^{-}}$ $N_{z, \mathrm{C}}$ molecules in the $x y$-plane, $z$-direction, and total, respectively. An effective Hamiltonian for this setup can be constructed as,

$$
H=H_{\mathrm{D}}+H_{\mathrm{A}}+H_{\mathrm{P}}+H_{\mathrm{DA}}+H_{\mathrm{DP}}+H_{\mathrm{AP}} .
$$

The term $H_{\mathrm{C}}=H_{\mathrm{C}}^{(\mathrm{sys})}+H_{\mathrm{C}}^{(\mathrm{B})}+H_{\mathrm{C}}^{(\mathrm{sys}-\mathrm{B})}$ is the Hamiltonian for the slab with the $\mathrm{C}$ chromophores, where (denoting $\hbar$ as the reduced Planck constant)

Table 1 Comparison of different cases of PARET arising from SC to donors and/or acceptors

SC to $\quad$ Features

Donors only

Acceptors only

Donors and acceptors
- PARET from donor polariton states; dominated by PRET contribution - Rate of EET from donor dark states $\approx$ bare (i.e., no SPs) FRET rate

- "Förster regime" of PARET

- Low EET to acceptor polariton states due to their low density of states (compared to dark states) and delocalized character

- Rate of EET to acceptor dark states $\approx$ bare FRET rate

- "Carnival effect": acceptor and donor reverse roles

- "Förster regime" of PARET

- Polariton states are delocalized across donors and acceptors

- Rate of PARET from polariton to dark states $\gg$ rate of PARET from dark/polariton to polariton states due to relative density of final states; dark-state manifolds are dense and act as traps

- PARET mediated by vibrational relaxation

- "Davydov/Redfield regime" of PARET 

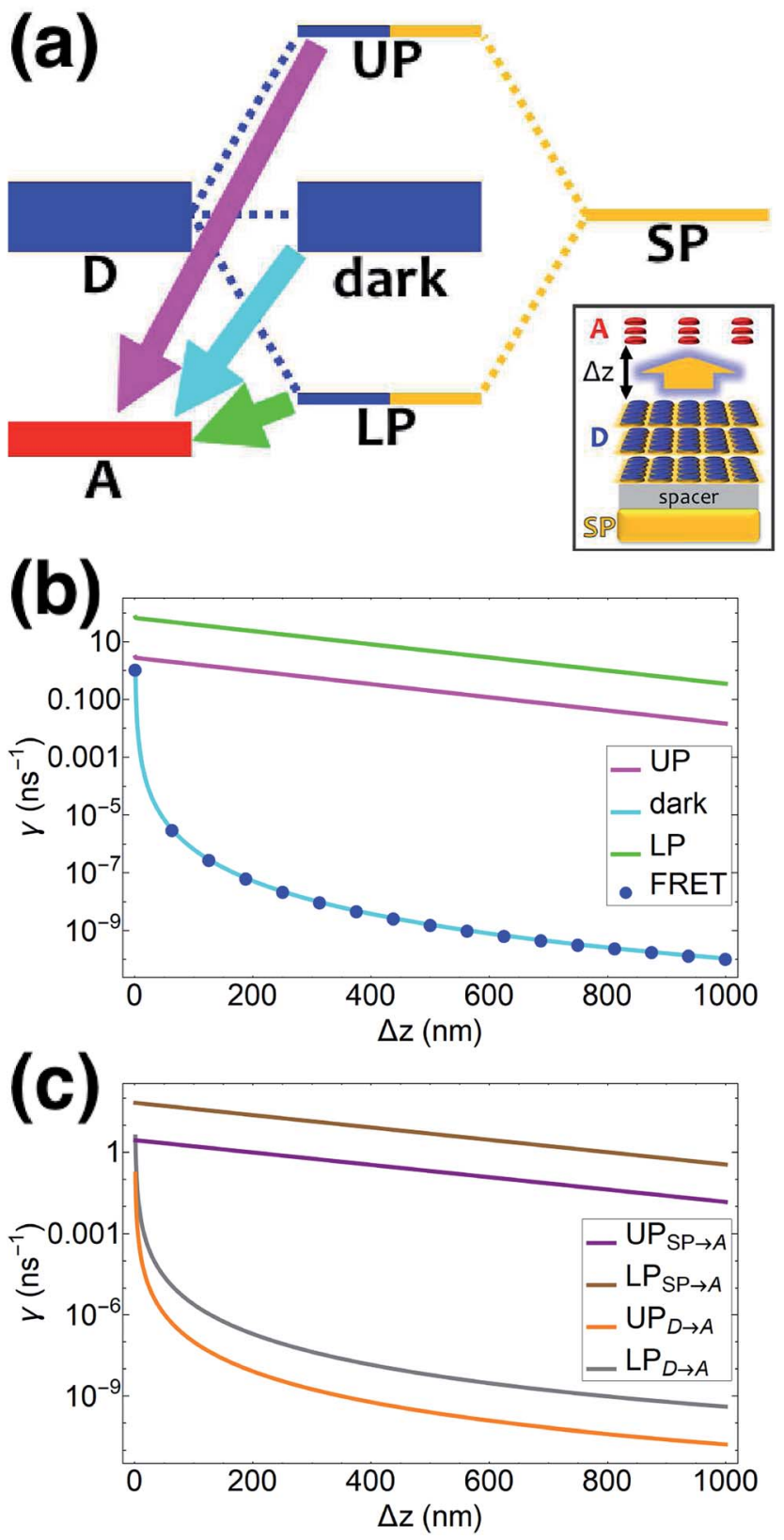

Fig. 1 (a) Schematic energy-level diagram showing the EET transitions from donors strongly coupled to SPs to bare acceptors. The thickness of the horizontal lines denotes the density of states while the thickness of arrows corresponds to rate of transition (thicknesses not drawn to scale). Inset: representation of the EET process from a thick and dense slab of donors (featuring SC to SPs) to a dilute monolayer of acceptors. (b) Rates as a function of donor-acceptor separation $\Delta z$ for EET from donor polariton and dark states to acceptors (lines). The rate from dark states and for the bare-donor FRET (dots), are calculated in the same manner. (c) Contributions of rates for transfer from donor UP and LP to acceptor states due to donor-acceptor (FRET) and SP-acceptor (PRET) interactions (see eqn (12a) and immediately preceding discussion).

$$
H_{\mathrm{C}}^{(\mathrm{sys})}=\hbar \omega_{\mathrm{C}} \sum_{i, j}\left|\mathrm{C}_{i j}\right\rangle\left\langle\mathrm{C}_{i j}\right|
$$

$$
\begin{gathered}
H_{\mathrm{C}}^{(\mathrm{B})}=\sum_{i, j} \sum_{q} \hbar \omega_{q, \mathrm{C}} b_{q, \mathrm{C}_{i j}}^{\dagger} b_{q, \mathrm{C}_{i j}}, \\
H_{\mathrm{C}}^{\text {(sys-B) }}=\sum_{i, j}\left|\mathrm{C}_{i j}\right\rangle\left\langle\mathrm{C}_{i j}\right| \sum_{q} \lambda_{q, \mathrm{C}} \hbar \omega_{q, \mathrm{C}}\left(b_{q, \mathrm{C}_{i j}}^{\dagger}+\text { h.c. }\right),
\end{gathered}
$$

represent the system (excitonic), bath (phononic), and systembath-coupling contributions, respectively. The label $\mathrm{C}_{i j}$ refers to a C exciton located at the $(i, j)$-th molecule of the corresponding slab $[(i, j)$ indexes an $(x y, z)$ coordinate $]$. We take every $\mathrm{C}_{i j}$ exciton to have energy $\hbar \omega_{\mathrm{C}}$ and neglect inter-site coupling since the latter provides an insignificant contribution to polariton dispersion when compared to the SP dispersion. Specifically, within the wavevector range of interest, inter-site coupling only induces a constant energy shift (which we assume to be included in $\omega_{\mathrm{C}}$ ) to the exciton subsystem. Thus, the exciton dispersion is only relevant at much shorter wavelengths. $b_{q, \mathrm{C}_{i j}}^{\dagger}\left(b_{q, \mathrm{C}_{i j}}\right)$ labels the creation (annihilation) of a phonon of energy $\hbar \omega_{q, \mathrm{C}}$ at the $q$-th vibrational mode of the $(i, j)$-th molecule in the C slab. Given the molecular character of the problem, vibronic coupling is assumed to be local ${ }^{30}$ (in contrast to that for relatively ordered materials such as crystals ${ }^{31}$ ): exciton $\mathrm{C}_{i j}$ couples linearly to $b_{q, \mathrm{C}_{i j}}^{\dagger}$ and $b_{q, \mathrm{C}_{i j}}$ but not to modes in other molecules; $;^{32}$ these couplings are characterized by Huang-Rhys factors $\lambda_{q, \mathrm{C}}{ }^{2} \cdot{ }^{33}$ The SP Hamiltonian $H_{\mathrm{P}}=H_{\mathrm{P}}^{\text {(sys) }}+H_{\mathrm{P}}^{(\mathrm{B})}+H^{(\text {sys }-}$ ${ }_{\mathrm{P}}^{\mathrm{B}}{ }_{\mathrm{P}}$ has similar form: $:^{34,35}$

$$
\begin{gathered}
H_{\mathrm{P}}^{(\mathrm{sys})}=\sum_{\vec{k}} \hbar \omega_{\vec{k}} a_{\vec{k}}^{\dagger} a_{\vec{k}}, \\
H_{\mathrm{P}}^{(\mathrm{B})}=\sum_{q} \hbar \omega_{q, \mathrm{P}} b_{q, \mathrm{P}}^{\dagger} b_{q, \mathrm{P}}, \\
H_{\mathrm{P}}^{\text {(sys-B) }}=\sum_{\vec{k}} \sum_{q} g_{q, \vec{k}}\left(b_{q, \mathrm{P}}^{\dagger} a_{\vec{k}}+\text { h.c. }\right),
\end{gathered}
$$

where $a_{\vec{k}}^{\dagger}\left(a_{\vec{k}}\right)$ labels the creation (annihilation) of an SP of energy $\hbar \omega_{\vec{k}}$ and in-plane wavevector $\vec{k}$. Bath modes indexed by $q, \mathrm{P}$ with corresponding operator $b_{q, \mathrm{P}}^{\dagger}\left(b_{q, \mathrm{P}}\right)$ and energy $\hbar \omega_{q, \mathrm{P}}$ are coupled to each SP mode $\vec{k}$ with strength $g_{q, \vec{k}}$. Specifically, these SP interactions occur with either electromagnetic or phonon modes and represent radiative and ohmic losses, respectively. ${ }^{35}$ The remaining rightmost terms in eqn (1) represent the dipoledipole interactions amongst donors, acceptors, and SP modes. The $H_{\mathrm{DA}}$ term is given by the electrostatic (near-field) dipoledipole interactions between donors and acceptors,

$$
H_{\mathrm{DA}}=\sum_{i, j} \sum_{l, m} \frac{\mu_{\mathrm{D}} \mu_{\mathrm{A}} \kappa_{i j l m}}{r_{i j l m^{3}}}\left(\left|\mathrm{~A}_{l m}\right\rangle\left\langle\mathrm{D}_{i j}\right|+\text { h.c. }\right),
$$

where $\mu_{\mathrm{C}}=\left|\vec{\mu}_{\mathrm{C}_{i j}}\right|$ for transition dipole moment (TDM) $\vec{\mu}_{\mathrm{C}_{i j}}$ corresponding to $\mathrm{C}_{i j}, r_{i j l m}$ is the distance between $\mathrm{D}_{j i}$ and $\mathrm{A}_{l m}$, and $\kappa_{i j l m}=\hat{\mu}_{\mathrm{D}_{i j}} \hat{\mu}_{\mathrm{A}_{l m}}-3\left(\hat{\mu}_{\mathrm{D}_{i j}} \hat{r}_{i j l m}\right)\left(\hat{\mu}_{\mathrm{A}_{l m}} \cdot \hat{r}_{i j l m}\right)$ is the orientational dependence of the interaction (we denote $\hat{v}$ as the unit vector corresponding to $\vec{v}$ ). We have ignored the corrections to $\kappa_{i j l m}$ due to reflected waves from the metal-despite their prominent effects in phenomena such as photoluminescence ${ }^{36}$ - since they are numerically involved and do not significantly change the order of magnitude of the bare dipole-dipole interaction; ${ }^{37}$ 
furthermore their expected effects in $H_{\mathrm{DA}}$ will be overwhelmed by $H_{\mathrm{CP}}$, as we shall explain in Sections 2.1 and 3. For simplicity, we take the permittivity on top of the metal to be a real-valued positive dielectric constant $\varepsilon_{\mathrm{d}}$. The light-matter interaction for species $\mathrm{C}$ in the rotating-wave approximation ${ }^{38}$ is also dipolar in nature and is described by ${ }^{39}$

$$
H_{\mathrm{CP}}=\sum_{i, j} \sum_{\vec{k}} \mu_{\mathrm{C}} \kappa_{\vec{k}} \mathrm{C}_{i j} \sqrt{\frac{\hbar \omega_{\vec{k}}}{2 \varepsilon_{0} S L_{\vec{k}}}} \mathrm{e}^{-a_{d \vec{k}} \mathrm{z}_{j, \mathrm{C}}} \mathrm{e}^{i \vec{k} \cdot \vec{R}_{i, \mathrm{C}}}\left|\mathrm{C}_{i j}\right\rangle\langle\mathrm{G}| a_{\vec{k}}+\text { h.c. }
$$

where $\left(\vec{R}_{i, \mathrm{C}}, \mathrm{Z}_{j, \mathrm{C}}\right)$ are the position coordinates of $\mathrm{C}_{i j}$ and $|\mathrm{G}\rangle$ is the electronic ground state (i.e., with no excitons). Just like in $H_{\mathrm{DA}}$, the interaction between an SP mode and a chromophore (indexed by $\vec{k}$ and $\mathrm{C}_{i j}$, respectively) has an orientation-dependent parameter $\quad \kappa_{\vec{k} \mathrm{C}_{i j}}=-\hat{\mu}_{\mathrm{C}_{i j}} \cdot\left(\hat{k}+i \frac{\vec{k}}{a_{d \vec{k}}} \hat{z}\right), \quad$ where $a_{d \vec{k}}=\sqrt{|\vec{k}|^{2}-\varepsilon_{d}\left(\omega_{\vec{k}} / c\right)^{2}}$ is the real-valued evanescent SP decay constant for the region above the metal. The light-matter coupling also includes the quantization length $L_{\vec{k}}$ (ref. 40) and area $S$ of the SP. We refer the reader to ESI Section $\mathrm{S} 1 \uparrow$ for further details of these terms.

The general Hamiltonian in eqn (1) describes a complex many-body problem consisting of excitons, SPs, and vibrations, all coupled with each other. To obtain physical insight on the opportunities afforded by this physical setup, we consider in the next sections two limit cases where either one or both types of chromophores are strongly coupled to the SP. The study of these two situations already provides considerable perspective on the wealth of novel EET phenomena hosted by this polaritonic system.

\subsection{Case (i): SC to only one chromophore C}

We consider the case where one of the chromophores C (D or A) is strongly coupled to an SP but the other, $\mathrm{C}^{\prime}$, is not. This can happen when the concentration or thickness of the $\mathrm{C}$ slab is sufficiently high and that of the $\mathrm{C}^{\prime}$ slab low. Under these circumstances, we write eqn (1) as $H=H_{0}^{(\mathrm{i})}+V^{(\mathrm{i})}$, where we define the zeroth-order Hamiltonian as $H_{0}^{(\mathrm{i})}=H_{\text {sys }}^{(\mathrm{i})}+H_{\mathrm{B}}+H_{\text {sys-B }}$. The system, bath, and their coupling are respectively characterized by $H_{\mathrm{sys}}^{(\mathrm{i})}=H_{\mathrm{D}}^{(\mathrm{sys})}+H_{\mathrm{A}}^{\text {(sys) }}+H_{\mathrm{P}}^{(\mathrm{sys})}+H_{\mathrm{CP}}, H_{\mathrm{B}}=H_{\mathrm{D}}^{(\mathrm{B})}+H_{\mathrm{A}}^{(\mathrm{B})}+$ $H_{\mathrm{P}}^{(\mathrm{B})}$, and $H_{\text {sys- }}=H_{\mathrm{D}}^{(\text {sys- })}+H_{\mathrm{A}}^{\text {(sys-B) }}+H_{\mathrm{P}}^{\text {(sys-B) }}$. The perturbation describing the weak interaction between chromophore $\mathrm{C}^{\prime}$ and the SC species is $V^{(\mathrm{i})}=H_{\mathrm{DA}}+H_{\mathrm{C}^{\prime} \mathrm{P}}$, i.e., the electrostatic interaction as in Förster ${ }^{1}$ theory. ${ }^{25,26}$ To diagonalize $H_{\text {sys }}^{(\mathrm{i})}$, we introduce a collective exciton basis comprised of bright $\mathrm{C}$ states with in-plane momenta matching those of the SP modes and ignore the very off-resonant SP-exciton couplings beyond the first Brillouin zone (FBZ) of the molecular system: ${ }^{\mathbf{3 9 4 1}}$ $H_{\text {sys }}^{(\mathrm{i})}=H_{\mathrm{C}^{\prime}}+\sum_{\vec{k} \in \mathrm{FBZ}} H_{\text {bright,C }}^{(\vec{k})}+H_{\text {dark,C }}+\sum_{\vec{k} \notin \mathrm{FBZ}} \hbar \omega_{\vec{k}} a_{\vec{k}}^{\dagger} a_{\vec{k}}$, where

$$
H_{\text {bright,C }}^{(\vec{k})}=\hbar \omega_{\mathrm{C}}\left|\mathrm{C}_{\vec{k}}\right\rangle\left\langle\mathrm{C}_{\vec{k}}\right|+\hbar \omega_{\vec{k}} a_{\vec{k}}^{\dagger} a_{\vec{k}}+g_{\mathrm{C}}(\vec{k})\left(\left|\mathrm{C}_{\vec{k}}\right\rangle\langle\mathrm{G}| a_{\vec{k}}+\text { h.c. }\right),
$$

$$
H_{\text {dark, }}=H_{\mathrm{C}}^{(\text {sys })}-\sum_{\vec{k} \in \mathrm{FBZ}} \hbar \omega_{\mathrm{C}}\left|\mathrm{C}_{\vec{k}}\right\rangle\left\langle\mathrm{C}_{\vec{k}}\right| .
$$

For each $\vec{k}$-mode in the FBZ, there is only one "bright" collective exciton state $\left|C_{\vec{k}}\right\rangle=\frac{1}{g_{\mathrm{C}}(\vec{k})} \sum_{i, j} \mu_{\mathrm{C}} \kappa_{\vec{k}} \mathrm{C}_{i j} \sqrt{\frac{\hbar \omega_{\vec{k}}}{2 \varepsilon_{0} S L_{\vec{k}}}} \mathrm{e}^{-a_{d \vec{k}} \vec{z}_{j, \mathrm{C}}} \mathrm{e}^{i \vec{k} \cdot \vec{k}_{i, \mathrm{C}}}\left|\mathrm{C}_{i j}\right\rangle$ that couples to the $\vec{k}$-th SP mode $|\vec{k}\rangle=a_{\vec{k}}^{\dagger}|0\rangle$, where

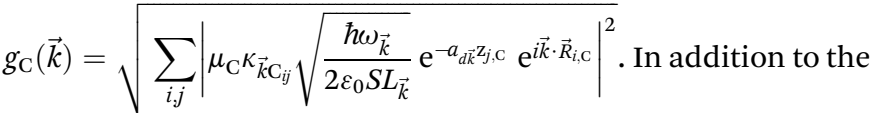
weakly coupled $\mathrm{C}^{\prime}$ states, $H_{\text {sys }}^{(\mathrm{i})}$ has two polariton eigenstates $\left|\alpha_{\mathrm{C}, \vec{k}}\right\rangle=c_{\mathrm{C}_{\vec{k}} \alpha_{\mathrm{C}, \vec{k}}}\left|\mathrm{C}_{\vec{k}}\right\rangle+c_{\vec{k} \alpha_{\mathrm{C}, \vec{k}}}|\vec{k}\rangle$ for $\alpha=\mathrm{UP}, \mathrm{LP}$ (upper polariton and lower polariton, respectively), which are also eigenstates of $H_{\text {bright,C }}^{(\vec{k})}$ for each $\vec{k} \in \mathrm{FBZ}$; throughout this work, $c_{m n}=\langle m \mid n\rangle$. Furthermore, there is a large reservoir of $N_{\mathrm{C}}-N_{x y, \mathrm{C}}=N_{x y, \mathrm{C}}\left(N_{z, \mathrm{C}}\right.$ - 1) "dark" (purely excitonic) eigenstates $\left|d_{\mathrm{C}, \vec{k}}\right\rangle\left(d=0,1, \ldots, N_{z, \mathrm{C}}\right.$ - 2) which are also eigenstates of $H_{\text {dark,C }}$ with bare chromophore energy $\hbar \omega_{\mathrm{C}}$.

EET rates between $\mathrm{C}$ and uncoupled $\mathrm{C}^{\prime}$ states can be derived by applying Fermi's golden rule; the corresponding perturbation $V^{(i)}$ connects vibronic-polariton eigenstates of $H_{0}^{(i)}$ as in FRET and MC-FRET theories.,42-44 For simplicity, we also invoke weak system-bath coupling $H_{\text {sys-B }}$, from which the following expression can be obtained, ${ }^{\mathbf{4 5 , 4 6}}$

$$
\gamma_{\mathrm{F} \leftarrow \mathrm{I}} \approx \frac{2 \pi}{\hbar}\left|\left\langle\mathrm{F}\left|V^{(i)}\right| \mathrm{I}\right\rangle\right|^{2} J_{\mathrm{F}, \mathrm{I}}
$$

This is the rate of transfer between $H_{\text {sys }}^{(\mathrm{i})}$ eigenstates $|\mathrm{I}\rangle$ and $|\mathrm{F}\rangle$, where $J_{\mathrm{F}, \mathrm{I}}$ is the spectral overlap between absorption and emission spectra, which depend on $H_{\mathrm{B}}$ and $H_{\text {sys-B }}$ (see Section $\mathrm{S} 2.1 \uparrow$ for derivation of $\gamma_{\mathrm{F} \leftarrow \mathrm{I}}$ and expression for $\left.J_{\mathrm{F}, \mathrm{I}}\right)$. Since our focus is to understand the general timescales expected for the PARET problem, in Section 3 we treat the broadening of electronic/polaritonic levels due to $H_{\text {sys-B }}$ as Lorentzian, although more sophisticated lineshape theories can be utilized if needed. ${ }^{33}$ Furthermore, we can in principle also refine eqn (7) to consider the complexities of vibronic mixing between the various eigenstates of $H_{\text {sys }}^{(i)}$, as done in recent works by Jang and Cao., ${ }^{3,42-44}$

It follows from eqn (7) that the rates from donor stateseither polaritons with given wavevector $\vec{k}$ or a uniform mixture of dark states with occupation $p_{\mathrm{D}_{\vec{k}}}=\frac{1}{N_{\mathrm{D}}-N_{x y, \mathrm{D}}}$ for all $d, \vec{k}$ - to the incoherent set of all bare acceptor states are,

$$
\begin{gathered}
\gamma_{\mathrm{A} \leftarrow \alpha_{\mathrm{D}, \vec{k}}}=\frac{2 \pi}{\hbar} \sum_{l, m}\left|\left\langle\mathrm{~A}_{l m}\left|H_{\mathrm{DA}}+H_{\mathrm{AP}}\right| \alpha_{\mathrm{D}, \vec{k}}\right\rangle\right|^{2} J_{\mathrm{A}, \alpha_{\mathrm{D}, \vec{k}}}, \\
\gamma_{\mathrm{A} \leftarrow \operatorname{dark}_{\mathrm{D}}}=\frac{2 \pi}{\hbar\left(N_{\mathrm{D}}-N_{x y, \mathrm{D}}\right)} \sum_{l, m} \sum_{\vec{k} \in \mathrm{FBZ}} \sum_{d}\left|\left\langle\mathrm{~A}_{l m}\left|H_{\mathrm{DA}}\right| d_{\mathrm{D}, \vec{k}}\right\rangle\right|^{2} J_{\mathrm{A}, d_{\mathrm{D}, \vec{k}}}
\end{gathered}
$$

Here, we notice that $\gamma_{\mathrm{A} \leftarrow \alpha_{\mathrm{D}, \vec{k}}}$ in eqn (8a) can be enhanced or suppressed relative to bare (in the absence of metal) FRET due to additional SP-resonance energy transfer ${ }^{47}$ (PRET) channel 
given by $H_{\mathrm{AP}}$, as well as the spectral overlap $J_{\mathrm{A} \leftarrow \alpha_{\mathrm{D}, \vec{k}}}$ that can be modified by tuning the energy of $\left|\alpha_{\mathrm{D}, \vec{k}}\right\rangle$. Similar findings were obtained for electron transfer with only donors strongly coupled to a cavity mode ${ }^{48}$ Given that $\left|\alpha_{\mathrm{D}, \vec{k}}\right\rangle$ corresponds to a delocalized state, one would expect a superradiant enhancement of the rate; $;^{5,6}$ in practice, this effect is minor due to the distance dependence of $H_{\mathrm{DA}}$ (see Section S2.3†). On the other hand, eqn (8b) presents an average rate $\gamma_{\mathrm{A} \leftarrow \text { dark }_{\mathrm{D}}}$ from the dark states and hence does not feature a PRET term. In fact, it converges (see Section S2.5 $\dagger$ ) to the bare FRET rate (eqn (12b) below) in the limit of large $N_{\mathrm{D}} \gg N_{x y, \mathrm{D}}$ (when there are many layers of chromophores along $z$ ) and isotropically averaged and orientationally uncorrelated TDMs $\vec{\mu}_{\mathrm{C}_{i j}}$ for both C $=\mathrm{D}$, A.

In contrast, strongly coupling the acceptor states to SPs yields the following rates:

$$
\begin{aligned}
& \gamma_{\alpha_{\mathrm{A}} \leftarrow \mathrm{D}}=\frac{2 \pi}{\hbar N_{\mathrm{D}}} \sum_{\vec{k} \in \mathrm{FBZ}} \sum_{i, j}\left|\left\langle\alpha_{\mathrm{A}, \vec{k}}\left|H_{\mathrm{DA}}+H_{\mathrm{DP}}\right| \mathrm{D}_{i j}\right\rangle\right|^{2} J_{\alpha_{\mathrm{A}, \vec{k}} \mathrm{D}}, \\
& \gamma_{\mathrm{dark}_{\mathrm{A}} \leftarrow \mathrm{D}}=\frac{2 \pi}{\hbar N_{\mathrm{D}}} \sum_{\vec{k} \in \mathrm{FBZ}} \sum_{d} \sum_{i, j}\left|\left\langle d_{\mathrm{A}, \vec{k}}\left|H_{\mathrm{DA}}+H_{\mathrm{DP}}\right| \mathrm{D}_{i j}\right\rangle\right|^{2} J_{d_{\mathrm{A}, \vec{k}} \mathrm{D}} .
\end{aligned}
$$

Here, we have calculated average rates over the $N_{\mathrm{D}}$ possible initial states at the D slab and summed over all final states for each polariton/dark band. Given the asymmetry of Fermi's golden rule with respect to initial and final states (rates scale with the probabilities of occupation of initial states and with the density of final states), ${ }^{5}$ the physical consequences of eqn (9) are quite different to those of its counterpart in eqn (8) when $N_{\mathrm{A}} \gg$ $N_{x y, \mathrm{~A}}$ and all TDMs are isotropically averaged and feature no orientational correlations amongst them. Seemingly counterintuitive in light of the various recently reported phenomena which are enhanced upon exciton delocalization, ${ }^{57}$ the rate of EET to polariton states is reduced substantially compared to the bare FRET rate (at short donor-acceptor separations; see Section S2.7 for a formal derivation of this statement). ${ }^{49}$ Nevertheless, this statement is easily understood from a finaldensity-of-states argument (see eqn (9a)): only $N_{x y, \mathrm{~A}}$ bright acceptor collective modes contribute to $\alpha \delta_{\mathrm{A} \leftarrow \mathrm{D}}$, whereas $N_{\mathrm{A}}$ localized acceptor states contribute to the bare FRET rate. On the other hand, $\gamma_{\text {dark }_{A} \leftarrow \mathrm{D}}$ behaves similarly to eqn ( $\left.8 \mathrm{~b}\right)$ in that it converges (see eqn $(13 \mathrm{~b})$ and $(\mathrm{S} 30 \mathrm{~b}) \dagger$ ) to the bare FRET rate. Thus, at donor-acceptor separations where the square of the FRET coupling exceeds on average that for PRET, the inequality $\gamma_{\alpha_{A} \leftarrow D} \ll \gamma_{\text {dark }_{A} \leftarrow D}$ is expected to hold.

Our analyses of eqn (8) and (9) reveal one of the main conclusions of this letter: while strongly coupling to D but not to A might yield a significant $D \rightarrow A$ EET rate change with respect to the bare case, strongly coupling to A but not to $D$ will change that process in a negligible manner. Interestingly, these trends have also been observed for transfer between layers of donor and acceptor quantum dots selectively coupled to metal nanoparticles in the weak-interaction regime. ${ }^{50}$ However, polariton formation with A is not useless, for one may consider the intriguing prospect of converting A states into new donors. As we shall show in the next paragraphs, this role reversal or "carnival effect" can be achieved when the UP is higher in energy than the bare donor states. These findings are quite general and should apply to other molecular processes as long as the interactions between reactants and products (taking the roles of donors and acceptors) also decay at large distances, a scenario that is chemically ubiquitous. ${ }^{51}$

\subsection{Case (ii): SC to both donor and acceptor chromophores}

We next consider the SC of SPs to both donors and acceptors. We rewrite eqn (1) as $H=H_{0}^{(i i)}+V^{(i i)}$, where $H_{0}^{(i i)}=H_{\text {sys }}^{(\text {ii) }}+H_{\mathrm{B}}$ and the perturbation is $V^{(\mathrm{ii})}=H_{\text {sys-B }}$ for interslab distance large enough to neglect interaction $H_{\mathrm{DA}}$ in $V^{(\mathrm{ii})}$. Here, $H_{\mathrm{sys}}^{(\mathrm{ii})}=H_{\mathrm{D}}^{\text {(sys) }}+$ $H_{\mathrm{A}}^{\text {(sys) }}+H_{\mathrm{DP}}+H_{\mathrm{AP}}+H_{\mathrm{DA}}+H_{\mathrm{P}}$ is the polariton Hamiltonian. As in the Davydov model, ${ }^{27,28}$ the EET pathways of interest become those where $H_{\text {sys-B }}$ induces vibrationally mediated relaxation (faster than dipole-dipole coupling in typical organic chromophores separated by 1-10 nm (ref. 2)) among the delocalized states resulting from SC. ${ }^{25,26}$ The transfer rates describing these processes can be deduced by Fermi's golden rule too, the resulting expressions coinciding with those derived with Redfield ${ }^{29}$ theory. ${ }^{25,26,52}$ Although not necessary, we take $N_{x y, \mathrm{D}}=N_{x y, \mathrm{~A}}$ $=N_{x y}$ to avoid mathematical technicalities about working with two $\vec{k}$ grids of different sizes, a complication that does not give more insight into the physics of interest. As done in Section 2.1, we rewrite $H_{\text {sys }}^{(\mathrm{ii})}$ in $\vec{k}$-space: $H_{\mathrm{sys}}^{(\mathrm{ii})}=\sum_{\vec{k} \in \mathrm{FBZ}} H_{\text {bright }}^{(\vec{k})}+H_{\mathrm{dark}, \mathrm{D}}+H_{\mathrm{dark}, \mathrm{A}}+H_{\mathrm{DA}}+\sum_{\vec{k} \notin \mathrm{FBZ}} \hbar \omega_{\vec{k}} a_{\vec{k}}^{\dagger} a_{\vec{k}}$,

where

$$
\begin{aligned}
H_{\text {bright }}^{(\vec{k})}= & \hbar \omega_{\mathrm{D}}\left|\mathrm{D}_{\vec{k}}\right\rangle\left\langle\mathrm{D}_{\vec{k}}\left|+\hbar \omega_{\mathrm{A}}\right| \mathrm{A}_{\vec{k}}\right\rangle\left\langle\mathrm{A}_{\vec{k}}\right|+\hbar \omega_{\vec{k}} a_{\vec{k}}^{\dagger} a_{\vec{k}} \\
& +g_{\mathrm{D}}(\vec{k})\left(\left|\mathrm{D}_{\vec{k}}\right\rangle\langle\mathrm{G}| a_{\vec{k}}+\text { h.c. }\right) \\
& +g_{\mathrm{A}}(\vec{k})\left(\left|\mathrm{A}_{\vec{k}}\right\rangle\langle\mathrm{G}| a_{\vec{k}}+\text { h.c. }\right),
\end{aligned}
$$

and the terms labeled dark are defined analogously to those in eqn (6b). For each $\vec{k} \in \mathrm{FBZ}$, there are three polariton eigenstates of $H_{\text {bright }}^{(\vec{k})}$ that are linear combinations of $\left|\mathrm{D}_{\vec{k}}\right\rangle,\left|\mathrm{A}_{\vec{k}}\right\rangle$, and $|\vec{k}\rangle$, and we call them UP, middle polariton (MP), and LP, according to their energy ordering. In addition, the presence of $H_{\mathrm{dark}, \mathrm{C}}$ yields $N_{\mathrm{C}}-N_{x y, \mathrm{C}}$ dark C eigenstates.

The resulting expressions for the rates of transfer from a single polariton state or average dark state to an entire polariton or dark state bands are

$$
\begin{aligned}
& \gamma_{\beta \leftarrow \alpha_{\vec{k}}}=\sum_{\vec{k}^{\prime} \in \mathrm{FBZ}} \sum_{\mathrm{C}}\left|c_{\mathrm{C}_{\vec{k}^{\prime}} \vec{\beta}_{\vec{k}^{\prime}}}\right|^{2}\left|c_{\mathrm{C}_{\vec{k}} \alpha_{\vec{k}}}\right|^{2} \sum_{i, j}\left|c_{C_{i j} \mathrm{C}_{\vec{k}^{\prime}}}\right|^{2}\left|c_{\mathrm{C}_{i j} \mathrm{C}_{\vec{k}}}\right|^{2} \mathscr{R}_{\mathrm{C}}\left(\omega_{\vec{\beta}_{\vec{k}^{\prime}} \alpha_{\vec{k}}}\right) \text {, } \\
& \gamma_{\alpha \leftarrow \text { dark }}=\frac{1}{N_{\mathrm{C}}-N_{x y}} \sum_{\vec{k}^{\prime} \in \mathrm{FBZ} \vec{k} \in \mathrm{FBZ}} \sum_{d}\left|c_{\mathrm{C}_{\vec{k}^{\prime}} \alpha_{\bar{k}^{\prime}}}\right|^{2} \\
& \times \sum_{i, j}\left|c_{C_{i j} C_{\overrightarrow{k^{\prime}}}}\right|^{2}\left|c_{\mathrm{C}_{i j} d_{\mathrm{C}, \vec{k}}}\right|^{2} \mathscr{R}_{\mathrm{C}}\left(\omega_{\tilde{\vec{k}}^{\prime}} \mathrm{C}\right), \\
& \gamma_{\text {dark }_{\mathrm{C}} \leftarrow \alpha_{\vec{k}}}=\sum_{\vec{k} \vec{k}^{\prime} \in \mathrm{FBZ} d} \sum_{d}\left|c_{\mathrm{C}_{\vec{k}} \alpha_{\mathrm{C}, \vec{k}}}\right| \sum_{i, j}\left|c_{\mathrm{C}_{i j} d_{\mathrm{C}, \vec{k}}}\right|^{2}\left|c_{\mathrm{C}_{i j} \mathrm{C}_{\vec{k}}}\right|^{2} \mathscr{R}_{\mathrm{C}}\left(\omega_{\mathrm{C} \alpha_{\vec{k}}}\right),
\end{aligned}
$$


for $\alpha, \beta=\mathrm{UP}, \mathrm{MP}, \mathrm{LP}$ and $\mathrm{C}=\mathrm{D}$, A (see Section $\mathrm{S} 3.1 \dagger$ for derivation of eqn (11)). To intuitively understand eqn (11a), note that $\left|c_{\mathrm{C}_{\vec{k}} \alpha_{\vec{k}}}\right|^{2}\left|c_{\mathrm{C}_{i j} \mathrm{C}_{\vec{k}}}\right|^{2}$ and $\left|c_{\mathrm{C}_{\vec{k}^{\prime}} \beta_{\vec{k}^{\prime}}}\right|^{2}\left|c_{\mathrm{C}_{i j} \mathrm{C}_{\vec{k}^{\prime}}}\right|^{2}$ are the fractions of exciton $\left|C_{i j}\right\rangle$ in the polariton states $\left|\alpha_{\vec{k}}\right\rangle$ and $\left|\beta_{\vec{k}^{\prime}}\right\rangle$, respectively, while $\mathrm{R}_{\mathrm{C}}\left(\omega_{{\overrightarrow{\vec{k}^{\prime}}}^{\prime} \alpha_{\vec{k}}}\right)$ is the single-molecule rate of vibrational relaxation at the energy difference $\omega_{\beta_{\vec{k}^{\prime}}}-\omega_{\alpha_{\vec{k}}}$. More specifically, $\mathrm{R}_{\mathrm{C}}(\omega)=2 \pi \Theta(-\omega)[n(-\omega)+1] \mathrm{J}_{\mathrm{C}}(-\omega)+2 \pi \Theta(\omega) n(\omega) \mathrm{J}_{\mathrm{C}}(\omega)$, where $\Theta(\omega)$ is the Heaviside step function, $n(\omega)=\frac{1}{\mathrm{e}^{\hbar \omega / k_{\mathrm{B}} T}-1}$ is the Bose-Einstein distribution function $\left(k_{\mathrm{B}}\right.$ is the Boltzmann constant and $T$ is temperature) for zero chemical potential $\mu=$ 0 , and $\mathscr{J}_{\mathrm{C}}(\omega)=\sum_{q} \lambda_{q, \mathrm{C}^{2}} \omega_{q, \mathrm{C}^{2}} \delta\left(\omega-\omega_{q, \mathrm{C}}\right)$ is the spectral density for chromophore $\mathrm{C}^{52}$ Hence, one can interpret $\beta \leftarrow \alpha_{\vec{k}}$ as a sum of incoherent processes (over $\mathrm{C}$ and $i, j$ ) where the (local) vibrational modes in $\mathrm{C}_{i j}$ absorb or emit phonons concomitantly inducing population transfer between the various eigenstates of $H_{\text {sys }}^{(\mathrm{ii})}$ Eqn (11b) and (11c) can be interpreted in a similar light. As an aside, we note that these transitions are analogous to the socalled intraband relaxation among collective exciton states in Jaggregates. ${ }^{53}$ We now comment on some important qualitative trends in these rates while for simplicity assuming that $N_{z, \mathrm{D}}=$ $N_{z, \mathrm{~A}}=N_{z}$. First, EET from polariton or dark states to a polariton band (eqn (11a) and (11b)) scale as $\frac{\mathrm{R}_{\mathrm{C}}}{N_{z}}$. To see this, note that both $\left|c_{\mathrm{C}_{\vec{k}^{\prime}} \beta_{\vec{k}^{\prime}}}\right|^{2}$ and $\left|c_{\mathrm{C}_{\vec{k}} \alpha_{\vec{k}}}\right|^{2}$ are $O(1)$, while $\left|c_{\mathrm{C}_{i j} \mathrm{C}_{\vec{k}^{\prime}}}\right|^{2}$ and $\left|c_{\mathrm{C}_{i j} \mathrm{C}_{\vec{k}}}\right|^{2}$ are $O\left(\frac{1}{N_{x y} N_{z}}\right)$, but the summations $\sum_{\vec{k} \in \mathrm{FBZ}}$ and $\sum_{i, j}$ are respectively carried over $N_{x y}$ and $N_{x y} N_{z}$ terms. On the other hand, $\gamma_{d a r k_{C} \leftarrow \alpha_{\vec{k}}}$ takes values that are on the order of the single-molecule decay $\mathrm{R}_{\mathrm{C}}\left(\omega_{\mathrm{C} \alpha_{\vec{k}}}\right)$. For sufficiently large $N_{z}$, these scalings are consistent with previous studies on relaxation dynamics of polaritons ${ }^{54-56}$ and can be summarized as follows: the dominant channels of relaxation are from the polariton states to a reservoir of dark states that share the same exciton character; their timescales are comparable to those of the corresponding singlechromophore vibrational relaxation; given the large density of states in this reservoir compared to the polariton bands, the dark states act as a population sink or trap from which population can only leak out very slowly. ${ }^{56,57}$

\section{Application of the theory}

The theory above is now applied to study EET kinetics associated with slabs of chromophores with $\hbar \omega_{\mathrm{D}}=2.1 \mathrm{eV}, \hbar \omega_{\mathrm{A}}=$ $1.88 \mathrm{eV}$; these transition energies are chosen to match those of the J-aggregated cyanine dyes (TDBC and BRK5714, respectively) used in previous polariton experiments. ${ }^{18,58}$ For simplicity, this section assumes $T=0$ and thus only considers downhill transfers to/from polariton and dark states. We describe the metal with Drude permittivity of silver $\left(\omega_{\mathrm{P}}=9.0 \mathrm{eV}\right.$, $\varepsilon_{\infty}=1 ;{ }^{59}$ see Section $\mathrm{S} 1 \dagger$ ) and all media at $\mathrm{z}>0$ (including molecular slabs) with $\varepsilon_{d}=1$. We model spectral overlaps (eqn (8) and (9)) with Lorentzian functions
$J_{\mathrm{F}, \mathrm{I}}=\frac{\frac{\Gamma_{\mathrm{I}}+\Gamma_{\mathrm{F}}}{2}}{\pi\left[\left(\frac{\Gamma_{\mathrm{I}}+\Gamma_{\mathrm{F}}}{2}\right)^{2}+\left(\hbar \omega_{\mathrm{FI}}\right)^{2}\right]}$ whose parameters are estimated as in Section S2.2; † we set $\Gamma_{\mathrm{A}} \approx \Gamma_{\mathrm{D}}=47 \mathrm{meV}$ to represent observed values for absorption of $\operatorname{TDBC}^{60}$ and $\Gamma_{P, \vec{k}}=\frac{v_{\mathrm{g}}(\vec{k})}{L_{\vec{k}}},{ }^{39}$ where $v_{\mathrm{g}}(\vec{k})$ is the SP group velocity. Rigorous treatments of lineshape functions have been previously reported in MC-FRET literature and could be applied to this problem as well, ${ }^{\mathbf{4 3 , 4 4 , 6 1 , 6 2}}$ although this effort is beyond the scope of our work. We also neglect differences in TDMs and assign $\mu_{\mathrm{D}}=\mu_{\mathrm{A}}=10 \mathrm{D}$, a typical number for cyanine dyes. ${ }^{63}$

We now proceed to simulations for Case (i), where only one of the molecular species forms polaritons. For simplicity, we assume isotropically oriented and spatially uncorrelated dipoles, upon which we find the interesting observation that the transfer rates in eqn (8) can be approximately decomposed into incoherent sums of FRET and PRET rates (see Sections S2.4 and $\mathrm{S} 2.5 \dagger$ for more explicit expressions, derivations, and justification of validity),

$$
\begin{aligned}
& \gamma_{\mathrm{A} \leftarrow \alpha_{\mathrm{D}, \vec{k}}} \approx \frac{2 \pi}{\hbar} \sum_{l} \sum_{i, j}\left(\left|c_{\mathrm{D}_{\vec{k}} \alpha_{\mathrm{D}, \vec{k}}}\right|^{2}\left|c_{\mathrm{D}_{i j} \mathrm{D} \vec{k}}\right|^{2}\left|\left\langle\mathrm{~A}_{l 0}\left|H_{\mathrm{DA}}\right| \mathrm{D}_{i j}\right\rangle\right|^{2}\right. \\
& \left.+\left|c_{\vec{k} \alpha_{\mathrm{D}, \vec{k}}}\right|^{2}\left|\left\langle\mathrm{~A}_{l 0}\left|H_{\mathrm{AP}}\right| \vec{k}\right\rangle\right|^{2}\right) J_{\mathrm{A}, \alpha_{\mathrm{D}, \vec{k}}} \\
& \gamma_{\mathrm{A} \leftarrow \text { dark }_{\mathrm{D}}}=\gamma_{\text {bare FRET }}=\frac{2 \pi}{\hbar N_{\mathrm{D}}} \sum_{l} \sum_{i, j}\left|\left\langle\mathrm{~A}_{l 0}\left|H_{\mathrm{DA}}\right| \mathrm{D}_{i j}\right\rangle\right|^{2} J_{\mathrm{A}, \mathrm{D}},
\end{aligned}
$$

where, as explained above, only $\gamma_{\mathrm{A} \leftarrow \alpha_{\mathrm{D}, \vec{k}}}$ differs from $\gamma_{\text {bare FRET }}$. More concretely, we consider a $35 \mathrm{~nm}$-thick slab of donors with $1 \times 10^{9}$ molecules per $\mu \mathrm{m}^{3}$ on top of a $1 \mathrm{~nm}$ spacer placed on a plasmonic metal film. We set the monolayer slab of acceptors with $1 \times 10^{4}$ molecules per $\mu \mathrm{m}^{2}$ at varying distances from the donors (Fig. 1a). Then the collective couplings of the donorresonant SP mode $\left(|\vec{k}|=1.1 \times 10^{7} \mathrm{~m}^{-1}\right)$ to donors and acceptors is $g_{\mathrm{D}}(\vec{k})=155 \mathrm{meV}$ and $g_{\mathrm{A}}(\vec{k}) \leq 2.5 \mathrm{meV}$, respectively. When there is no separation between donor and acceptor slabs, rates $>1 \mathrm{~ns}^{-1}$ (Fig. 1b) are obtained for transfer to acceptors from the UP $\left(\sim 10 \mathrm{~ns}^{-1}\right)$, LP $\left(\sim 100 \mathrm{~ns}^{-1}\right)$, or the set of dark states $(\sim 10$ $\left.n s^{-1}\right)$. As separation increases however, the rate from dark states decays much faster than those from either UP or LP. This difference stems from the slowly decaying PRET contribution of the polaritons, as well as the totally excitonic character of the dark states, which can only undergo FRET but not PRET (Fig. 1a and b). In fact, for large distances, the FRET contribution becomes significantly overwhelmed by PRET (Fig. 1c), in consistency with previous studies in the weak SP-coupling regime. ${ }^{64}$ As the distance between slabs approaches $1 \mu \mathrm{m}$, it is fascinating that while transfer from dark states (and thus bare FRET) practically vanishes, the rate from either LP $\left(\sim 1 \mathrm{~ns}^{-1}\right)$ or UP $\left(\sim 0.01 \mathrm{~ns}^{-1}\right)$ is still at or above typical fluorescence decay rates (0.01-10 ns $\left.{ }^{-1}\right) .{ }^{65}$ Roughly speaking (in FRET language), this PARET has a Förster distance in the $\mu \mathrm{m}$ range, or 1000-fold greater than the typical nm-range. ${ }^{66}$ Interestingly, the LP rate exceeds the UP one by 1-2 orders of magnitude at all 
(a)
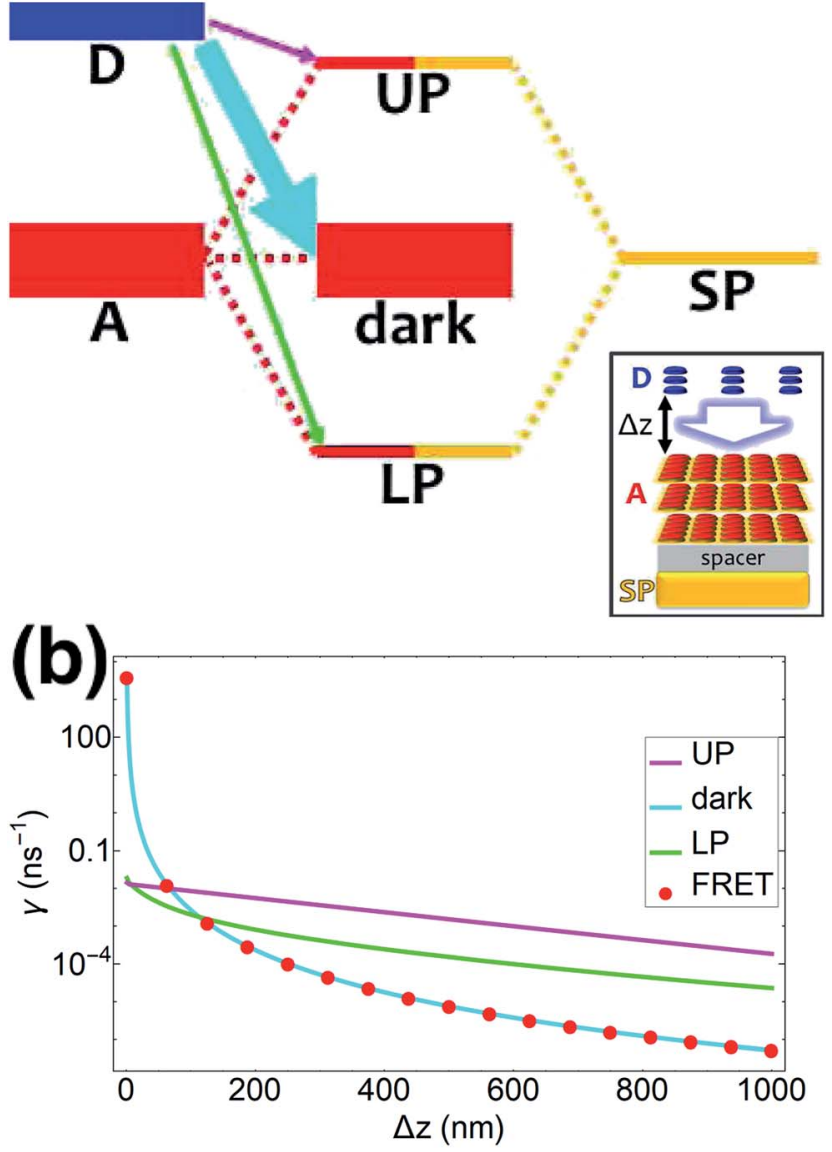

Fig. 2 (a) Schematic energy-level diagram showing the EET transitions from bare donors to acceptors strongly coupled to SPs. The thickness of the horizontal lines denotes the density of states while the thickness of arrows corresponds to rate of transition (thicknesses not drawn to scale). Inset: representation of the EET process from a dilute monolayer of donors to a thick and dense slab of acceptors (featuring SC to SPs). (b) Rates as a function of donor-acceptor separation $\Delta z$ for energy transfer from donors to acceptor polaritons and dark states (lines). The rate to dark states and for bare-acceptor FRET (dots), are calculated in the same manner.

separations due to greater spectral overlap with the acceptor (Fig. 1a and $\mathrm{S} 1 \dagger$ ).

In contrast, strongly coupling the acceptors to a resonant SP mode does not lead to the aforementioned PARET from donors to acceptors (Fig. 2). Making the same assumptions as above, i.e., taking isotropically oriented and spatially uncorrelated dipoles, we obtain (Section S2.7†),

$$
\begin{aligned}
& \gamma_{\alpha_{\mathrm{A}} \leftarrow \mathrm{D}} \approx \frac{2 \pi}{\hbar N_{\mathrm{D}}} \sum_{\vec{k} \in \mathrm{FBZ}} \sum_{i}\left(\sum_{l, m}\left|c_{\mathrm{A}_{\vec{k}}-\alpha_{\mathrm{A}, \vec{k}}}\right|^{2}\left|c_{\mathrm{A}_{l m} \mathrm{~A}_{\vec{k}}}\right|^{2}\left|\left\langle\mathrm{~A}_{l m}\left|H_{\mathrm{DA}}\right| \mathrm{D}_{i 0}\right\rangle\right|^{2}\right. \\
& \left.+\left|c_{\vec{k} \alpha_{\mathrm{A}, \vec{k}} \mid}\right|^{2}\left|\left\langle\vec{k}\left|H_{\mathrm{DP}}\right| \mathrm{D}_{i 0}\right\rangle\right|^{2}\right) J_{\alpha_{\mathrm{A}, \vec{k}}, \mathrm{D}}
\end{aligned}
$$

$$
\gamma_{\text {dark }_{\mathrm{A}} \leftarrow \mathrm{D}}=\gamma_{\text {bare FRET }}^{\prime}=\frac{2 \pi}{\hbar N_{\mathrm{D}}} \sum_{i} \sum_{l, m}\left|\left\langle\mathrm{~A}_{l m}\left|H_{\mathrm{DA}}\right| \mathrm{D}_{i 0}\right\rangle\right|^{2} J_{\mathrm{A}, \mathrm{D}}
$$

We consider (Fig. 2a) a 50 nm-thick acceptor slab with a concentration of $1 \times 10^{9}$ molecules per $\mu \mathrm{m}^{3}$ on top of the $1 \mathrm{~nm}$ spacer placed on the metal, and a monolayer of donors with concentration $1 \times 10^{4}$ molecules per $\mu \mathrm{m}^{2}$ at varying distances from the acceptors. Notice that $\gamma_{\mathrm{dark}_{\mathrm{A}} \leftarrow \mathrm{D}}$ becomes another bare FRET rate like in eqn (12b). For $\gamma_{\alpha_{A} \leftarrow D}$, we see that PRET still dominates over FRET for long distances (Fig. S2 $\dagger$ ). However, due to the suppression of $\gamma_{\alpha_{A} \leftarrow \mathrm{D}}$ relative to $\gamma_{\mathrm{dark}_{\mathrm{A}} \leftarrow \mathrm{D}}$ explained in Section 2.1, the limited spatial range of interactions of $H_{\mathrm{DA}}$ and $H_{\mathrm{DP}}$, and the fact that the donor energy is lower than that of $\left|\mathrm{UP}_{\mathrm{A}, \vec{k}}\right\rangle$ for most $\vec{k} \in \mathrm{FBZ}$ (Fig. S3 $\dagger$ ), the rates to the acceptor UP band fall below fluorescence timescales ${ }^{65}$ and therefore offer no

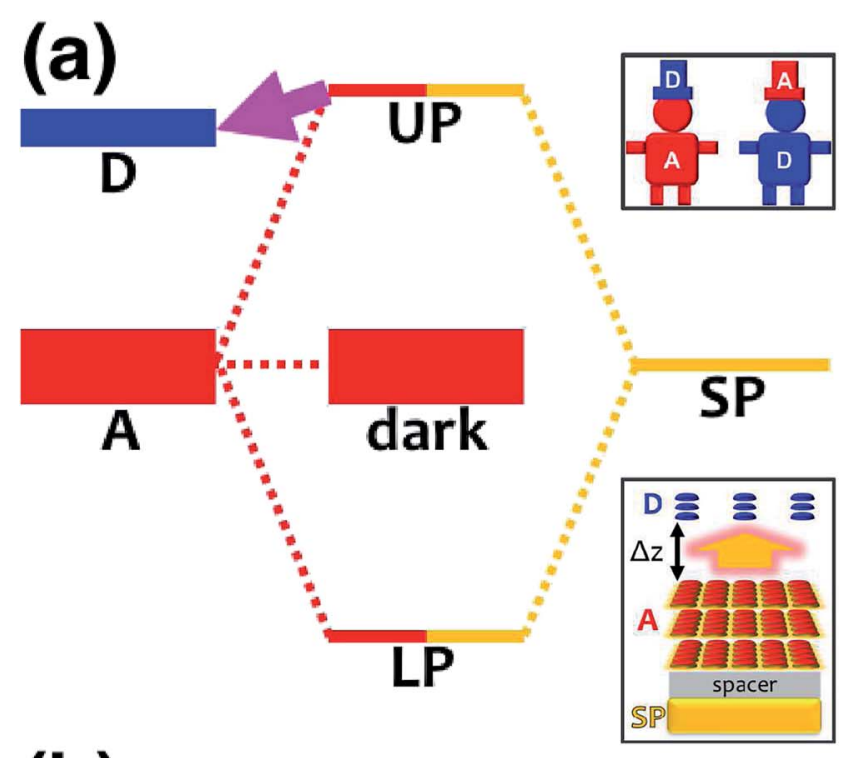

\section{(b)}

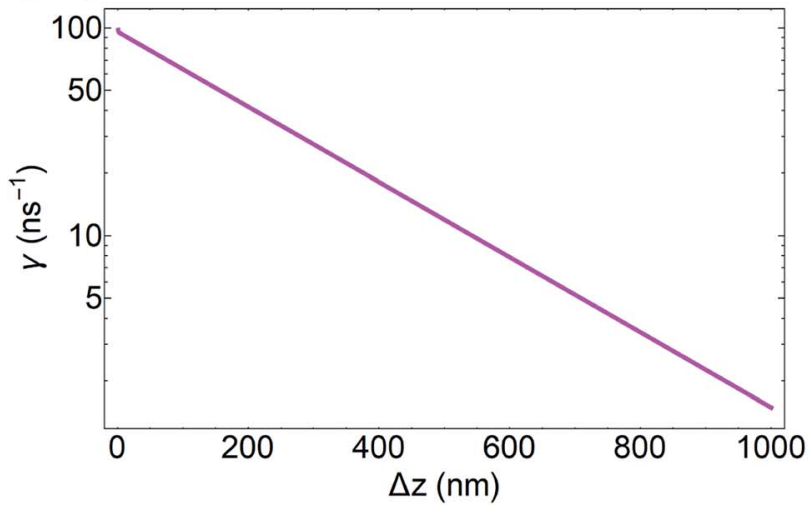

Fig. 3 (a) Schematic energy-level diagram showing the "carnivaleffect"-EET role-reversal process from acceptor UP state to bare donors. Insets: (top) cartoon illustrating the "carnival effect" between donors and acceptors and (bottom) representation of the reversedrole EET process from a thick and dense slab of acceptors (featuring SC to SPs) to a dilute monolayer of donors. (b) Rate as a function of donor-acceptor separation $\Delta z$ for energy transfer from acceptor UP to bare donors. 
meaningful enhancements with respect to the bare FRET case (Fig. 2b). While the first two reasons also explain the similarly low rates to the LP band, the major factor is that most states $\left|\mathrm{LP}_{\mathrm{A}, \vec{k}}\right\rangle$ essentially overlap only with acceptors that are closer to the metal-and thus farther from the donors-due to the evanescent nature of the SP-exciton coupling (eqn (5)).

Coupling SPs to acceptors need not, however, be a disappointment. Increasing the acceptor slab thickness to $140 \mathrm{~nm}$ affords for the acceptor-resonant SP mode $\left(|\vec{k}|=9.8 \times 10^{6} \mathrm{~m}^{-1}\right)$ the collective coupling $g_{\mathrm{A}}(\vec{k})=237 \mathrm{meV}$ while keeping $g_{\mathrm{D}}(\vec{k})=$ $1.7 \mathrm{meV}$. Consequently, the acceptor UP energy $\hbar \omega_{\mathrm{UP}_{A, \vec{k}}}$ is lifted higher than $\hbar \omega_{\mathrm{D}}$ (Fig. 3a and $\mathrm{S} 4 \dagger$ ), thus allowing for the carnival effect where the donors and acceptors reverse roles. Due to sufficient spectral overlap between the acceptor UP and donor states (Fig. 3a and $\mathrm{S} 4 \dagger$ ), transfer from UP occurs at $\sim 100 \mathrm{~ns}^{-1}$ for donor-acceptor separation of $1 \mathrm{~nm}$ and drops only to $\sim 1 \mathrm{~ns}^{-1}$ when this separation approaches $1 \mu \mathrm{m}$ (Fig. 3b). On the other hand, neither the acceptor dark nor LP states contribute to this reversed PARET given their lack of spectral overlap with the donors and detailed balance (especially at $T=0$ ). This result provides the second main conclusion of our work: polaritons offer great versatility to control spectral overlaps without actual chemical modifications to the molecules and can therefore endow them with new physical properties. Before proceeding to simulations for Case (ii), it should first be noted that while our model neglects intermediate- and far-field donor-acceptor dipoledipole interactions that become relevant at $\sim \mu \mathrm{m}$ distances, these couplings are expected to be small compared to PRET couplings and therefore should not qualitatively change our results for Case (i). The relative insignificance of the far-field, or radiative, contributions is consistent with their correspondence to emission followed by absorption (of a real photon). ${ }^{67}$ Regardless, these additional interactions can be modeled using a quantum electrodynamics treatment ${ }^{68}$ of energy transfer in bulk media according to previous literature. ${ }^{69}$ Second, we highlight that the PARET from polariton states for the donorsonly and reversed cases of SC may not be efficient in experiments due to its competition with vibrational relaxation from UP to dark states $(\sim 10-100$ fs for exciton-microcavity systems ${ }^{54,70-73}$ ) and/or radiative decay (dominated by fast cavity leakage $\sim 10-100$ fs for both microcavities ${ }^{72-74}$ and $\mathrm{SPs}^{75,76}$ ). However, conditions may be optimized to suppress these deleterious pathways by detuning the SP energy relative to that of the strongly coupled chromophore such that the polariton state is mostly excitonic and has an enhanced lifetime. ${ }^{77}$

Finally, when both chromophores are strongly coupled to SPs, we limit ourselves to donor-acceptor separations $>10 \mathrm{~nm}$ to ignore $H_{\mathrm{DA}}$ terms (an approximation discussed in Section 2.2 and validated by the calculations above demonstrating that FRET coupling is significantly diminished at such distances). The thickness $(35 \mathrm{~nm})$ and density $\left(1 \times 10^{9}\right.$ molecules per $\left.\mu \mathrm{m}^{3}\right)$ of each slab is large enough to allow for SC of the donor-resonant SP mode $\left(|\vec{k}|=1.1 \times 107 \mathrm{~m}^{-1}\right.$, gD $(\vec{k})=155 \mathrm{meV}$, and $\mathrm{gA}(\vec{k})$ $=142 \mathrm{meV}$ ) to both chromophores separated up to $\sim 400 \mathrm{~nm}$ (Fig. S6b $\dagger$ ). To evaluate the rates derived (Section S3.2†) from eqn (11) under the condition $N_{\mathrm{C}} \gg N_{x y, \mathrm{C}}$ for $\mathrm{C}=\mathrm{D}$, A, we introduce a spectral density representing intramolecular exciton-phonon coupling of TDBC: $\mathrm{J}_{\mathrm{A}}(\omega) \approx \mathrm{J}_{\mathrm{D}}(\omega)=\mathrm{J}(\omega)$, where

$$
\mathscr{J}(\omega)=\sum_{q \in B_{\mathrm{TDBC}}} \lambda_{q^{2}} \omega_{q^{2}} \frac{\frac{\Gamma / \hbar}{2}}{\pi\left[\left(\frac{\Gamma / \hbar}{2}\right)^{2}+\left(\omega-\omega_{q}\right)^{2}\right]},
$$

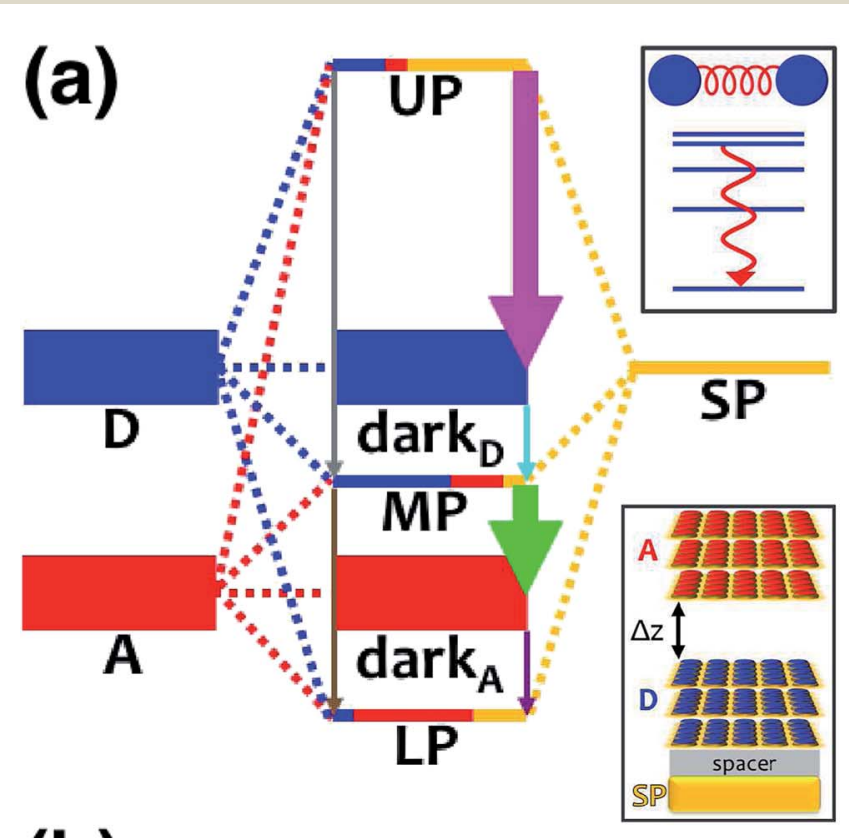

(b)
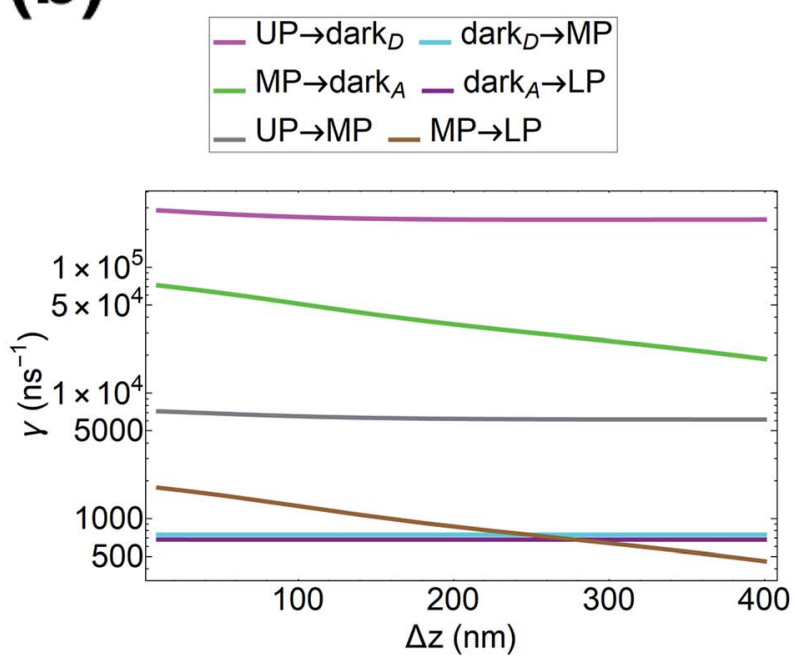

Fig. 4 (a) Schematic energy-level diagram showing the EET transitions among polariton and dark states for both donors and acceptors strongly coupled to SPs. The SP mode is resonant with the donor transition (for polariton as initial state); the donor slab lies $1 \mathrm{~nm}$ above the metal and has fixed position $(z>0)$ while the acceptor slab is moved in the $+z$-direction to vary the donor-acceptor separation $\Delta z$. The thickness of the horizontal lines denotes the density of states while the thickness of arrows corresponds to rate of transition (thicknesses not drawn to scale). Insets: (top) cartoon illustrating vibrational relaxation, the EET mechanism for this case of SC, and (bottom) representation of the setup of thick and dense slabs for both types of chromophores (featuring SC to SPs) for both types of chromophores. (b) Rates for selected downhill transitions as a function of $\Delta z$. 
$\Gamma=47 \mathrm{meV}$ is equal to the chromophore decay energy, and $B_{\text {TDBC }}$ is the discrete set (Section S3.2†) of localized vibrational modes which significantly couple to each TDBC exciton. Such coupling has been experimentally ${ }^{70-73,78}$ and theoretically ${ }^{54,55,79-81}$ supported as the mechanism of vibrational relaxation for the dye; our spectral density has been reconstructed from the works of Agranovich and coworkers. ${ }^{54,55}$ By placing the donor slab on top of the spacer on the metal and varying the acceptor position above the donors (Fig. 4a), we find that for all donor-acceptor separations, the rates of PARET from UP to dark donors $\left(\sim 10^{5}\right.$ $\left.\mathrm{ns}^{-1}\right)$ and MP to dark acceptors $\left(\sim 10^{4}-10^{5} \mathrm{~ns}^{-1}\right)$ are substantially higher compared to those from dark donors to MP $\left(\sim 10^{3}\right.$ $\left.\mathrm{ns}^{-1}\right)$ and dark acceptors to LP $\left(\sim 10^{3} \mathrm{~ns}^{-1}\right)$ (Fig. 4b). These observations are in agreement with our discussion above, where the dark state manifolds act as population sinks due to their high density of states. Indeed, we also notice that the rates for $\mathrm{UP} \rightarrow \operatorname{dark}_{\mathrm{D}}$ and MP $\rightarrow$ dark $_{\mathrm{A}}$ are enhanced (Fig. $4 \mathrm{~b}$ ) compared to those of UP $\rightarrow$ MP and MP $\rightarrow$ LP, respectively, by approximately $N_{z}=35$, the analytically estimated ratio solely based on the associated density of final states. Another interesting detail seen from Fig. $4 \mathrm{~b}$ is that the UP $\rightarrow$ MP (MP $\rightarrow$ LP) rate with respect to the interslab distance $\Delta z$ is essentially parallel to that of UP $\rightarrow \operatorname{dark}_{\mathrm{D}}$ (MP $\rightarrow \operatorname{dark}_{\mathrm{A}}$ ). This is a consequence of the MP (LP) essentially having donor (acceptor) energy and character for most points in the FBZ (see dispersion curve, Fig. S6†). The $\mathrm{UP} \rightarrow$ dark $_{\mathrm{A}}$ and UP $\rightarrow$ LP processes behave similarly (Fig. S5†).

These calculated rates establish consistency with a number of recent notable experiments. First, our results corroborate the experimental observation of efficient PARET for separated donor and acceptor slabs of cyanine dyes strongly coupled to a microcavity. ${ }^{18,19}$ While our SP model for the SC of both excitons cannot account for the exact distance-independent PARET $^{\mathbf{1 8}}$ between donor and acceptor slabs in a microcavity, the rates are essentially constant over hundreds of nanometers due to the slowly decaying SP fields. Additional validation of our theory is obtained by defining rate parameters $\mathrm{C}$ (functions of the $\gamma$ rates above, see Sections S3.3 and S3.4†) that can be directly compared to those experimentally reported (Table 2). ${ }^{\mathbf{1 9}}$

Table 2 Comparison between PARET rate parameters $\mathrm{C}$ for donor and acceptor cyanine dye J-aggregates strongly coupled to SP (theory) and microcavity (experiment) modes $^{a}$

\begin{tabular}{lll}
\hline $\mathrm{C}$ & $\mathrm{SP}$ (theory) $^{b}$ & $\begin{array}{l}\text { Microcavity } \\
\text { (experiment) }^{c}\end{array}$ \\
\hline $\mathrm{C}_{5}^{\mathrm{UPB}}$ & $(2.9-3.2 \mathrm{fs})^{-1}$ & $(34 \mathrm{fs})^{-1}$ \\
$\mathrm{C}_{2}$ & $(177-259 \mathrm{ps})^{-1}$ & $(603 \mathrm{ps})^{-1}$ \\
$\mathrm{C}_{5}^{\mathrm{MPB}}$ & $(6.9-12 \mathrm{fs})^{-1}$ & $(8.5 \mathrm{fs})^{-1}$ \\
$\mathrm{C}_{1}$ & $(178-243 \mathrm{ps})^{-1}$ & $(228 \mathrm{ps})^{-1}$
\end{tabular}

${ }^{a}$ See Sections S3.3 and S3.4 for additional details. ${ }^{b}$ Ranges of the rate parameters calculated from eqn (S45), plotted in Fig. S7, and accounting for the facts that typical polariton photoluminescence experiments occur at room temperature and probe only final polariton states near the anticrossings. ${ }^{22}{ }^{c}$ Rate parameters that were obtained from experimental fitting of a kinetic model and that describe the PARET processes for a blend of J-aggregating NK-2707 (donors) and TDBC (acceptors) cyanine dyes both strongly coupled to a microcavity mode. ${ }^{19}$
Since experimental $\mathrm{C}$ have not been determined for the setup of our work, we instead use those describing a blend of two cyanine dyes where physical separation of the dyes did not significantly change the observed photoluminescence. ${ }^{19}$ In our work, we obtained rates that assume zero temperature and sum across the whole polariton band in the FBZ; in practice, experiments occur at room temperature and probe polariton photoluminescence around a narrow window of wavevectors close to the anticrossings $\left(\sim 10^{7} \mathrm{~m}^{-1}\right.$ in (ref. 82)). If we take these experimental details into account when calculating $\mathrm{C}$, we notice good agreements with our theory (see Table 2). As an aside, we reiterate that there are other experimental subtleties, notably competing processes such as radiative decay, that we have not considered but may compromise the longevity of the polariton states and thereby influence the observation of the EET phenomena predicted throughout this work for the two cases of SC.

Given the significant differences between the microcavity $^{83,84}$ and ${ }^{8 P-b a s e d}{ }^{85}$ systems, let alone experimental uncertainty, the accordance between our theory and the aforementioned experiments highlights the remarkable robustness of cavity SC of donor and acceptor excitons as a method for PARET. Moreover, we have arrived at the third main conclusion of this paper: when donor and acceptors are both strongly coupled to a photonic mode, efficient energy exchange over hundreds of nm can occur via vibrational relaxation; more generally, local vibrational couplings can induce nonlocal transitions given sufficient delocalization of the polariton species-irrespective of spatial separation. Seemingly "spooky", this action at a (far) distance is a manifestation of donor-acceptor entanglement resulting from strong light-matter coupling. ${ }^{\mathbf{8 6}}$ While this relaxation mechanism and entanglement is present in typical molecular aggregates, ${ }^{87,88}$ the novelty in the polariton setup is the remarkable mesoscopic range of interactions that is effectively produced.

\section{Conclusions}

In summary, we have theoretically calculated experimentally consistent rates of PARET for various cases of SC. We employed a polariton (plexciton) setup consisting of a metal whose SP modes couple to donor and/or acceptor chromophores. For the case where a single type of chromophore is strongly coupled to SPs, we have demonstrated that energy transfer starting from delocalized states can be enhanced due to increased spectral overlap compared to the bare FRET case. Astonishingly, this transfer can remain fast up to $1 \mu \mathrm{m}$ due to slowly decaying PRET coupling with respect to metal-chromophore separation when compared to the faster decaying interchromophoric dipoledipole coupling. Also, we have shown that delocalizing the acceptors is a poor strategy to enhance EET starting from the donors, but can lead to an intriguing and efficient role-reversed ("carnival-effect") EET starting from the acceptors. These observations shed new light on the timely debate of how to harness coherence to enhance molecular processes. ${ }^{49}$ Given their generality, they can also be applied to guide the design of polaritonic systems to control other chemical processes that have similar donor-acceptor flavor (e.g., charge transfer, ${ }^{89}$ 
electron transfer, ${ }^{48}$ singlet fission ${ }^{77}$ ) or reactant-product nature (e.g., cis-trans isomerization, ${ }^{\mathbf{4 1 , 9 0}}$ dissociation ${ }^{89}$ ). Finally, our calculated rates support vibrational relaxation as the mechanism of PARET when both donors and acceptors are strongly coupled to a cavity mode. The results obtained in this work affirm light-matter SC as a promising and novel means to engineer novel interactions between molecular systems across mesoscopic length scales, thus opening doors to remotecontrolled chemistry.

\section{Conflicts of interest}

There are no conflicts to declare.

\section{Acknowledgements}

M. D., R. F. R., and J. Y.-Z. acknowledge funding from the NSF CAREER Award CHE-164732. L. A. M.-M. was supported by the UC-Mexus CONACyT scholarship for doctoral studies. M. D., R. F. R., J. Y.-Z., and L. A. M.-M. are also grateful for generous UCSD startup funds. V. M. M. acknowledges funding from DOE BES through grant no. DE-SC0017760. M. D. thanks Jorge Campos-González-Angulo and Rahul Deshmukh for useful discussions.

\section{References}

1 T. Förster, Ann. Phys., 1948, 2, 55-75.

2 A. Govorov, P. L. H. Martínez and H. V. Demir, Understanding and Modeling Förster-type Resonance Energy Transfer (FRET): Introduction to FRET, Springer, Singapore, 2016.

3 S. Jang, M. D. Newton and R. J. Silbey, Phys. Rev. Lett., 2004, 92, 218301.

4 S. Jang, M. D. Newton and R. J. Silbey, J. Phys. Chem. B, 2007, 111, 6807-6814.

5 I. Kassal, J. Yuen-Zhou and S. Rahimi-Keshari, J. Phys. Chem. Lett., 2013, 4, 362-367.

6 S. Lloyd and M. Mohseni, New J. Phys., 2010, 12, 075020.

7 G. S. Engel, T. R. Calhoun, E. L. Read, T.-K. Ahn, T. Mančal, Y.-C. Cheng, R. E. Blankenship and G. R. Fleming, Nature, 2007, 446, 782.

8 S. Duque, P. Brumer and L. A. Pachón, Phys. Rev. Lett., 2015, 115, 110402.

9 G. D. Scholes, Annu. Rev. Phys. Chem., 2003, 54, 57-87.

10 A. T. Haedler, K. Kreger, A. Issac, B. Wittmann, M. Kivala, N. Hammer, J. Kohler, H.-W. Schmidt and R. Hildner, Nature, 2015, 523, 196-199.

11 S. K. Saikin, M. A. Shakirov, C. Kreisbeck, U. Peskin, Y. N. Proshin and A. Aspuru-Guzik, J. Phys. Chem. C, 2017, 121, 24994-25002.

12 L.-Y. Hsu, W. Ding and G. C. Schatz, J. Phys. Chem. Lett., 2017, 8, 2357-2367.

13 X. Zhang, C. A. Marocico, M. Lunz, V. A. Gerard, Y. K. Gunâko, V. Lesnyak, N. Gaponik, A. S. Susha, A. L. Rogach and A. L. Bradley, ACS Nano, 2014, 8, 12731283.
14 W. Ding, L.-Y. Hsu and G. C. Schatz, J. Chem. Phys., 2017, 146, 064109.

15 D. Bouchet, D. Cao, R. Carminati, Y. De Wilde and V. Krachmalnicoff, Phys. Rev. Lett., 2016, 116, 037401.

16 J. de Torres, P. Ferrand, G. Colas des Francs and J. Wenger, ACS Nano, 2016, 10, 3968-3976.

17 S. Götzinger, L. d. S. Menezes, A. Mazzei, S. Kühn, V. Sandoghdar and O. Benson, Nano Lett., 2006, 6, 11511154.

18 X. Zhong, T. Chervy, L. Zhang, A. Thomas, J. George, C. Genet, J. A. Hutchison and T. W. Ebbesen, Angew. Chem., Int. Ed., 2017, 56, 9034-9038.

19 D. M. Coles, N. Somaschi, P. Michetti, C. Clark, P. G. Lagoudakis, P. G. Savvidis and D. G. Lidzey, Nat. Mater., 2014, 13, 712-719.

20 M. Slootsky, X. Liu, V. M. Menon and S. R. Forrest, Phys. Rev. Lett., 2014, 112, 076401.

21 J. Feist and F. J. García-Vidal, Phys. Rev. Lett., 2015, 114, 196402.

22 J. Schachenmayer, C. Genes, E. Tignone and G. Pupillo, Phys. Rev. Lett., 2015, 114, 196403.

23 J. Yuen-Zhou, S. K. Saikin, T. Zhu, M. C. Onbasli, C. A. Ross, V. Bulovic and M. A. Baldo, Nat. Commun., 2016, 7, 11783.

24 D. M. Basko, F. Bassani, G. C. La Rocca and V. M. Agranovich, Phys. Rev. B: Condens. Matter Mater. Phys., 2000, 62, 15962-15977.

25 M. Yang and G. R. Fleming, Chem. Phys., 2002, 282, 163-180. 26 E. N. Zimanyi and R. J. Silbey, Philos. Trans. R. Soc., A, 2012, 370, 3620-3637.

27 A. S. Davydov, J. Exp. Theor. Phys., 1948, 18, 210-218.

28 A. Davydov, Theory of molecular excitons, McGraw-Hill, New York, 1962.

29 A. Redfield, in The theory of relaxation processes, Elsevier, 1965, vol. 1, pp. 1-32.

30 M. R. Philpott, J. Chem. Phys., 1970, 52, 5842-5850.

31 T. F. Soules and C. B. Duke, Phys. Rev. B: Solid State, 1971, 3, 262-274.

32 T. Holstein, Ann. Phys., 1959, 8, 325-342.

33 S. Mukamel, Principles of nonlinear optical spectroscopy, Oxford University Press, 1995.

34 D. Walls and G. Milburn, Quantum Optics, Springer-Verlag, 1994.

35 E. Waks and D. Sridharan, Phys. Rev. A: At., Mol., Opt. Phys., 2010, 82, 043845.

36 J. Yuen-Zhou, S. K. Saikin and V. Menon, ArXiv e-prints, arXiv: 1711.11213.

37 F. Zhou, Y. Liu and Z.-Y. Li, Opt. Lett., 2011, 36, 1969-1971. 38 M. Scully and M. Zubairy, Quantum Optics, Cambridge University Press, New York, New York, 1997.

39 A. González-Tudela, P. A. Huidobro, L. Martín-Moreno, C. Tejedor and F. J. García-Vidal, Phys. Rev. Lett., 2013, 110, 126801.

40 A. Archambault, F. Marquier, J.-J. Greffet and C. Arnold, Phys. Rev. B: Condens. Matter Mater. Phys., 2010, 82, 035411.

41 L. A. Martínez-Martínez, R. F. Ribeiro, J. A. Campos Gonzalez Angulo and J. Yuen-Zhou, ACS Photonics, 2017. 
42 S. Jang, Y. Jung and R. J. Silbey, Chem. Phys., 2002, 275, 319332.

43 S. Jang and R. J. Silbey, J. Chem. Phys., 2003, 118, 9312-9323. 44 J. Ma and J. Cao, J. Chem. Phys., 2015, 142, 094106.

45 S. Baghbanzadeh and I. Kassal, Phys. Chem. Chem. Phys., 2016, 18, 7459-7467.

46 S. Baghbanzadeh and I. Kassal, J. Phys. Chem. Lett., 2016, 7, 3804-3811.

47 G. L. Liu, Y.-T. Long, Y. Choi, T. Kang and L. P. Lee, Nat. Methods, 2007, 4, 1015.

48 F. Herrera and F. C. Spano, Phys. Rev. Lett., 2016, 116, 238301.

49 G. D. Scholes, G. R. Fleming, L. X. Chen, A. Aspuru-Guzik, A. Buchleitner, D. F. Coker, G. S. Engel, R. van Grondelle, A. Ishizaki, D. M. Jonas, J. S. Lundeen, J. K. McCusker, S. Mukamel, J. P. Ogilvie, A. Olaya-Castro, M. A. Ratner, F. C. Spano, K. B. Whaley and X. Zhu, Nature, 2017, 543, 647. 50 T. Ozel, P. L. Hernandez-Martinez, E. Mutlugun, O. Akin, S. Nizamoglu, I. O. Ozel, Q. Zhang, Q. Xiong and H. V. Demir, Nano Lett., 2013, 13, 3065-3072.

51 J. A. Hutchison, T. Schwartz, C. Genet, E. Devaux and T. W. Ebbesen, Angew. Chem., Int. Ed., 2012, 51, 1592-1596. 52 V. May and O. Kühn, Charge and Energy Transfer Dynamics in Molecular Systems, Wiley, 2011.

53 M. Bednarz, V. A. Malyshev and J. Knoester, J. Chem. Phys., 2002, 117, 6200-6213.

54 V. M. Agranovich, M. Litinskaia and D. G. Lidzey, Phys. Rev. B: Condens. Matter Mater. Phys., 2003, 67, 085311.

55 M. Litinskaya, P. Reineker and V. M. Agranovich, J. Lumin., 2004, 110, 364-372.

56 J. del Pino, J. Feist and F. J. Garcia-Vidal, New J. Phys., 2015, 17, 053040.

57 A. Canaguier-Durand, C. Genet, A. Lambrecht, T. W. Ebbesen and S. Reynaud, Eur. Phys. J. D, 2015, 69, 24.

58 X. Zhong, T. Chervy, S. Wang, J. George, A. Thomas, J. A. Hutchison, E. Devaux, C. Genet and T. W. Ebbesen, Angew. Chem., Int. Ed., 2016, 55, 6202-6206.

59 E. Palik, Handbook of Optical Constants of Solids II, Academic Press, 1985.

60 J. Bellessa, C. Bonnand, J. C. Plenet and J. Mugnier, Phys. Rev. Lett., 2004, 93, 036404.

61 J. Ma, J. Moix and J. Cao, J. Chem. Phys., 2015, 142, 094107. 62 J. M. Moix, J. Ma and J. Cao, J. Chem. Phys., 2015, 142, 094108.

63 S. Valleau, S. K. Saikin, M.-H. Yung and A. A. Guzik, J. Chem. Phys., 2012, 137, 034109.

64 A. O. Govorov, J. Lee and N. A. Kotov, Phys. Rev. B: Condens. Matter Mater. Phys., 2007, 76, 125308.

65 B. Valeur and M. Berberan-Santos, Molecular Fluorescence: Principles and Applications, Wiley, 2nd edn, 2012.

66 I. Medintz and N. Hildebrandt, FRET - Förster Resonance Energy Transfer: From Theory to Applications, Wiley, 2013.

67 D. L. Andrews, Chem. Phys., 1989, 135, 195-201.
68 G. Juzeliūnas and D. L. Andrews, Phys. Rev. B: Condens. Matter Mater. Phys., 1994, 49, 8751-8763.

69 H. T. Dung, L. Knöll and D.-G. Welsch, Phys. Rev. A: At., Mol., Opt. Phys., 2002, 66, 063810.

70 D. M. Coles, P. Michetti, C. Clark, W. C. Tsoi, A. M. Adawi, J.-S. Kim and D. G. Lidzey, Adv. Funct. Mater., 2011, 21, 3691-3696.

71 T. Virgili, D. Coles, A. M. Adawi, C. Clark, P. Michetti, S. K. Rajendran, D. Brida, D. Polli, G. Cerullo and D. G. Lidzey, Phys. Rev. B: Condens. Matter Mater. Phys, 2011, 83, 245309.

72 D. M. Coles, P. Michetti, C. Clark, A. M. Adawi and D. G. Lidzey, Phys. Rev. B: Condens. Matter Mater. Phys., 2011, 84, 205214.

73 N. Somaschi, L. Mouchliadis, D. Coles, I. E. Perakis, D. G. Lidzey, P. G. Lagoudakis and P. G. Savvidis, Appl. Phys. Lett., 2011, 99, 143303.

74 D. G. Lidzey, A. M. Fox, M. D. Rahn, M. S. Skolnick, V. M. Agranovich and S. Walker, Phys. Rev. B: Condens. Matter Mater. Phys., 2002, 65, 195312.

75 M. van Exter and A. Lagendijk, Phys. Rev. Lett., 1988, 60, 4952.

76 C. Sönnichsen, T. Franzl, T. Wilk, G. von Plessen, J. Feldmann, O. Wilson and P. Mulvaney, Phys. Rev. Lett., 2002, 88, 077402.

77 L. A. Martínez-Martínez, M. Du, R. F. Ribeiro, S. Kéna-Cohen and J. Yuen-Zhou, J. Phys. Chem. Lett., 2018, 9, 1951-1957.

78 D. M. Coles, R. T. Grant, D. G. Lidzey, C. Clark and P. G. Lagoudakis, Phys. Rev. B: Condens. Matter Mater. Phys., 2013, 88, 121303.

79 J. Chovan, I. E. Perakis, S. Ceccarelli and D. G. Lidzey, Phys. Rev. B: Condens. Matter Mater. Phys., 2008, 78, 045320.

80 P. Michetti and G. C. La Rocca, Phys. Rev. B: Condens. Matter Mater. Phys., 2008, 77, 195301.

81 P. Michetti and G. C. La Rocca, Phys. Rev. B: Condens. Matter Mater. Phys., 2009, 79, 035325.

82 T. K. Hakala, J. J. Toppari, A. Kuzyk, M. Pettersson, H. Tikkanen, H. Kunttu and P. Törmä, Phys. Rev. Lett., 2009, 103, 053602.

83 M. S. Skolnick, T. A. Fisher and D. M. Whittaker, Semicond. Sci. Technol., 1998, 13, 645.

84 R. J. Holmes and S. R. Forrest, Org. Electron., 2007, 8, 77-93. 85 P. Törmä and W. L. Barnes, Rep. Prog. Phys., 2015, 78, 013901.

86 F. J. Garcia-Vidal and J. Feist, Science, 2017, 357, 1357-1358. 87 V. Kenkre and P. Reineker, Exciton Dynamics in Molecular Crystals and Aggregates, Springer-Verlag, 1982.

88 M. Sarovar, A. Ishizaki, G. R. Fleming and K. B. Whaley, Nat. Phys., 2010, 6, 462-467.

89 J. Flick, M. Ruggenthaler, H. Appel and A. Rubio, Proc. Natl. Acad. Sci. U. S. A., 2017, 114, 3026-3034.

90 J. Galego, F. J. Garcia-Vidal and J. Feist, Nat. Commun., 2016, 7, 13841. 